\title{
TTR
}

Traduction, terminologie, re?daction

\section{Sherry Simon and Paul St-Pierre (eds.). Changing the Terms. Translating in the Postcolonial Era, Ottawa, University of Ottawa Press, 2000, 305 p.}

\section{Alexis Nouss}

Volume 16, numéro 1, 1er semestre 2003

Controverse en traductologie

Controversy in Translation Studies

URI : https://id.erudit.org/iderudit/008565ar

DOI : https://doi.org/10.7202/008565ar

Aller au sommaire du numéro

\section{Éditeur(s)}

Association canadienne de traductologie

ISSN

0835-8443 (imprimé)

1708-2188 (numérique)

Découvrir la revue

Citer ce compte rendu

Nouss, A. (2003). Compte rendu de [Sherry Simon and Paul St-Pierre (eds.).

Changing the Terms. Translating in the Postcolonial Era, Ottawa, University of Ottawa Press, 2000, 305 p.] TTR, 16(1), 246-249. https://doi.org/10.7202/008565ar

Tous droits réservés ( $\odot$ TTR: traduction, terminologie, rédaction — Les auteurs, Ce document est protégé par la loi sur le droit d'auteur. L’utilisation des d'utilisation que vous pouvez consulter en ligne.

https://apropos.erudit.org/fr/usagers/politique-dutilisation/ 
montrer des ouvertures dans la langue française pour recevoir le parler noir. Lavoie choisit de puiser dans la littérature antillaise afin de décentrer la langue française. C'est grâce aux œuvres des auteurs antillais Patrick Chamoiseau et Jacques Roumain, dont l'écriture fait preuve d'une véritable recherche stylistique et linguistique, qu'elle proposera une solution à la problématique de la traduction du Black English en français. Elle établira les liens littéraires, ainsi que la parenté socio-linguistique et historique, entre le français créolisé et le vernaculaire noir américain, en prenant bien soin de préciser qu'il ne s'agit pas là d'équivalents. Le français créolisé, grâce à un procédé de compensation, assumerait plutôt la même fonction identificatrice au sein du texte que le Black English. Ces pistes esquissées par Lavoie pourraient mener à une entreprise de retraduction qui donnerait enfin à lire l'œuvre de Twain, dans toute sa plurivocalité, au public français, afin de « réactiver à sa pleine mesure la charge profondément revendicatrice qu'elle véhicule». (p. 213). En définitive, cette étude constitue un apport indiscutable à la traductologie, et plus particulièrement à l'étude du sociolecte en traduction. Grâce à l'analyse minutieuse des traductions, l'auteure jette un éclairage nouveau sur l'œuvre de Mark Twain et en fait ressortir toute la richesse plurilinguistique. Au terme de la lecture de cet ouvrage et à la lumière des enjeux soulevés par la traduction française du vernaculaire noir américain, on aurait souhaité lire un extrait traduit par Lavoie mettant en pratique les principes énoncés dans le dernier chapitre, comme elle l'avait fait dans sa thèse de doctorat. Mais le lecteur devra plutôt attendre qu'un traducteur entreprenne cette tâche de retraduction monumentale pour enfin lire un Huckleberry Finn en français à la hauteur de l'original.

\section{Savoyane Henri-Lepage Université McGill}

Sherry Simon and Paul St-Pierre (eds.). Changing the Terms. Translating in the Postcolonial Era, Ottawa, University of Ottawa Press, 2000, 305 p.

Illustration de couverture : l'image tendre et charmante de deux mainates sur les branches d'un arbre, l'un rouge, l'autre bleu, l'un triste, l'autre malicieux. Celui du haut regarde vers l'Ouest, l'autre vers l'Est. Bien trouvé pour un ouvrage traitant du postcolonialisme. La curiosité pousse à chercher en page intérieure 
l'origine de l'illustration, une gravure faite à Calcutta au XIX siècle. Mais la légende précise : "Two mynahs ». "Two mynahs ", « deux mainates ». Est-cela, " Changing the terms » ? Évidemment non, ce ne serait qu'une théorie du transcodage, unanimement honni en traductologie. Alors qu'il faut ici observer que, pour désigner l'oiseau imitateur de la voix humaine - métaphore du bon ou du mauvais traducteur ? -, l'anglais a choisi de rester proche du mot malais original alors que le français francise.

" Changing the Terms " implique de changer le sémantisme des termes mêmes dans lesquels se comprend l'énoncé " Changing the Terms ». Il ne s'agit plus de modification ou de remplacement mais d'interaction et de négociation entre les termes visés. Un des apports du post-colonialisme est précisément d'avoir substitué à l'analyse classique du colonialisme en termes de rapports de pouvoir assymétriques et de logiques de domination une lecture insistant sur les modifications apportées par l'expérience coloniale aux deux cultures et aux deux identités en présence. Perspective nouvelle qui entraîne en traductologie l'abandon d'une conception linéaire et téléologique valorisant le texte d'arrivée aux dépens du texte de départ pour l'adoption d'une vision étudiant les rapports qui s'établissent entre les deux textualités. Le changement ne doit pas en rester au niveau de la saisie des objets et des acteurs sur la scène post-coloniale mais affecter également la méthodologie et l'idiome de la théorisation cette dernière notion à n'utiliser que dans la déclinaison « critique » de l'École de Francfort. Sur le modèle de la «relexification » que Chantal Zabus (The Post-Colonial Studies Reader, Routledge, 2001, pp. 314-318) substitue à la notion de traduction lorsqu'elle considère le travail des langues africaines dans les langues européennes, révélant et élaborant un troisième code, la théorie post-colonialiste doit mettre en ouvre une grille de lecture empruntant à la fois aux registres d'intellection occidentaux et aux systèmes conceptuels des cultures non-occidentales. À la question emblématique et tant influente, "Can the Subaltern speak ? », de G. Spivak, on adjoindra "Can the Subaltern Think ? ", compris bien entendu comme : "Peuvent-ils insérer leur pensée dans les cadres réflexifs en cours quant à la sphère académico-intellectuelle dominante? »

Un des grands mérites de ce recueil est d'offrir des exemples probants du changement de termes quant à la traductologie post-colonialiste, à savoir l'émergence d'une 
perspective qui renouvelle les présupposés mêmes de l'analyse traductologique. On citera les articles de Michael Cronin, Leo TakHung Chan, Jean-Marc Gouanvic, Michaela Wolf, Probal Dasgupta (sans doute le plus novateur). Ce n'est pas minimiser la valeur des autres articles mais simplement noter, par contraste, un certain classicisme de l'approche qui ne les empêche toutefois pas de fournir un excellent matériau d'étude pour l'élaboration d'une nouvelle traductologie. Car, épistémologiquement, c'est une meilleure connaissance de l'objet qui permet de refaçonner le champ d'étude; un changement de paradigme ne s'opère pas par le biais d'une volonté externe mais est appelé par l'insuffisance méthodologique révélée dans l'approfondissement du savoir. Le sous-titre de l'ouvrage, Translating in the Postcolonial Era, semble privilégier l'une des deux compréhensions possibles du postcolonialisme : une périodicité ou une herméneutique. L'alternative s'appliquait déjà au post-modernisme, déjouée lorsque se distinguent post-modernisme et post-modernité, le premier terme étant d'ordre théorique, le second d'ordre historique. On peut, au demeurant, pareillement opposer post-colonialisme et postcolonialité. Un volume tel que celui-ci offre l'occasion de juger la nature du post-colonialisme. N'est-il qu'un nouvel appareillage théorique unificateur et systémisant ou, de par sa nature, un discours critique défaisant les hégémonies conceptuelles, y compris lorsqu'elles se veulent émancipatrices ? En ce sens, la division bipartite du recueil — «(Post)colonialism and the Powers of translation " suivi de " Scenes of Negociation » — paraîtrait tendre à reproduire une dichotomie suspecte entre la théorie et la pratique. Mais les articles ne s'inscrivent pas tous dans l'orientation du soustitre qui les accueille et les auteurs pré-cités ne sont pas tous regroupés dans la première partie.

La prédominance indienne dans les corpus à l'étude n'induit aucunement un quelconque géocentrisme. Elle est précisément révélatrice du lien entre pratique et réflexion. Les spécificités de la traduction dans le contexte indien sont telles qu'elles permettent non la généralisation des résultats de l'étude, contraire au relativisme post-moderne, mais l'expérimentation de postures analytiques novatrices, ainsi que le montrent les articles de Jawharlal Nehru ou Paul St-Pierre. Les autres aires géographicoculturelles (Irlande, Chine, Caraïbes, Amérique latine, Québec) abordées autorisent une comparaison des contextes prévenant la réduction des problématiques de la traductologie post-coloniale à une rationalité universalisable. Cette souplesse pragmatique, en 
revanche, lui accorde une dimension hautement politique. Car "Changing the terms " n'est pas un énoncé s'auto-validant. L'administration Bush s'affaire à changer les termes de la politique et du droit internationaux, détruisant un équilibre minimal en vigueur depuis la Seconde Guerre mondiale. En matière de traduction, elle s'est surtout souciée, selon le journal Le Monde, de pourvoir ses soldats en terre irakienne d'ordinateurs portables aux logiciels aptes à traduire «Levez les mains » et « Rendez vous ». C'est dire que devant ce nouvel empire — car, comme l'a montré Toni Negri, la motivation ne relève pas de l'impérialisme mais d'un esprit impérial - , ce que peut penser la traductologie en interrogeant l'histoire coloniale revêt des implications politiques.

Sur fond de globalisation, entre les lectures concluant à l'homogénéisation et celles défendant les pratiques particularisantes, Sherry Simon choisit de reconnaître un dialogue entre le nationalisme culturel et l'hétérogénéité post-nationale. Les traducteurs, familiers de la tension entre la déterritorialisation universalisante et la reterritorialisation ethnocentrique, sont, à cette fin, de précieux acteurs. "Changing the terms of cultural transmission » leur revient, conclut-elle, là où une traductologie traditionnelle ne leur attribuait de fonction que de « changing the terms » entre deux langues. Regarder dans des directions opposées n'empêche pas les deux mainates de chanter ensemble.

\section{Alexis Nouss Université de Montréal}

\section{Michel Plourde avec la collaboration de Hélène Duval et de Pierre Georgeault (sous la dir.), Le Français au Québec. 400 ans d'histoire et de vie, Montréal, Fides, 2002, 516 p.}

Cet ouvrage réalisé sous l'égide du Conseil supérieur de la langue française (anciennement le Conseil de la langue française) est imposant à plusieurs égards. Rédigé par une équipe de plus de quatre-vingts spécialistes, il présente l'évolution linguistique en l'articulant de manière systématique et rigoureuse à l'histoire générale du Québec (démographique, politique, culturelle et sociale). Divisé en quatre grandes périodes (1608-1760, 17601850, 1850-1960, 1960-2000), il constitue une synthèse intelligente, neuve et pédagogique de l'évolution linguistique au Québec. La richesse de l'information est remarquable, en 